\title{
Fragmentation methods on the balance: unambiguous top-down mass spectrometric characterization of oxaliplatin-ubiquitin binding sites
}

\author{
Samuel M. Meier • Yury O. Tsybin • Paul J. Dyson • \\ Bernhard K. Keppler • Christian G. Hartinger
}

Received: 31 July 2011 /Revised: 19 October 2011 /Accepted: 19 October 2011 /Published online: 8 November 2011

(C) Springer-Verlag 2011

\begin{abstract}
The interaction between oxaliplatin and the model protein ubiquitin $(\mathrm{Ub})$ was investigated in a topdown approach by means of high-resolution electrospray ionization mass spectrometry (ESI-MS) using diverse tandem mass spectrometric (MS/MS) techniques, including collision-induced dissociation (CID), higher-energy C-trap dissociation (HCD), and electron transfer dissociation (ETD). To the best of our knowledge, this is the first time that metallodrug-protein adducts were analyzed for the metal-binding site by ETD-MS/MS, which outperformed both CID and HCD in terms of number of identified metallated peptide fragments in the mass spectra and the localization of the binding sites. Only ETD allowed the simultaneous and exact determination of Met1 and His68 residues as binding partners for oxaliplatin. CID-MS/MS experiments were carried out on orbitrap and ion cyclotron
\end{abstract}

Published in the $A N A K O N$ special issue with guest editors P. Dittrich, D. Günther, G. Hopfgartner, and R. Zenobi

Electronic supplementary material The online version of this article (doi:10.1007/s00216-011-5523-0) contains supplementary material, which is available to authorized users.

S. M. Meier · B. K. Keppler • C. G. Hartinger $(\bowtie)$

Institute of Inorganic Chemistry, University of Vienna,

Waehringer Str. 42,

1090 Vienna, Austria

e-mail: christian.hartinger@univie.ac.at

S. M. Meier • B. K. Keppler · C. G. Hartinger

Research Platform "Translational Cancer Therapy Research",

University of Vienna,

Waehringer Str. 42,

1090 Vienna, Austria

Y. O. Tsybin • P. J. Dyson • C. G. Hartinger

Institut des Sciences et Ingénierie Chimiques,

Ecole Polytechnique Fédérale de Lausanne (EPFL),

1015 Lausanne, Switzerland resonance (ICR)-FT mass spectrometers and both instruments yielded similar results with respect to number of metallated fragments and the localization of the binding sites. A comparison of the protein secondary structure with the intensities of peptide fragments generated by collisional activation of the $[\mathrm{Ub}+\mathrm{Pt}-(\mathrm{chxn})]$ adduct $[\mathrm{chxn}=(1 R, 2 R)$ cyclohexanediamine] revealed a correlation with cleavages in solution phase random coil areas, indicating that the $N$ terminal $\beta$-hairpin and $\alpha$-helix structures are retained in the gas phase.

Keywords Anticancer metallodrugs · Tandem mass spectrometry Electron transfer dissociation - Oxaliplatin . Ubiquitin

\section{Introduction}

Platinum-based chemotherapeutics such as cisplatin, carboplatin, and oxaliplatin play an important role in cancer treatment. To exert their therapeutic effects, platinum-based anticancer drugs primarily target cellular DNA [1]. However, prior to entering the cell, plasma proteins also constitute excellent binding partners and it was found that $\mathrm{Pt}(\mathrm{II})$-based metallodrugs bind to human serum albumin (HSA) and other plasma proteins upon administration [2, 3]. Furthermore, it is thought that the interaction with proteins accounts at least partially for the deactivation of these therapeutic agents and also for some adverse side effects observed during chemotherapy of cancer patients [4]. Understanding the binding to biomolecules is thus crucial for the development of novel metal-based anticancer drugs with enhanced selectivity and reduced side effects, however, the preferred binding sites of the Pt(II)-based anticancer drugs on proteins are still a matter of debate [5-8]. 
The identification of binding sites and of the nature of metallodrug-protein interactions both with clinically approved metal-based anticancer agents and also with developmental metallodrugs have been attempted using a wide range of bioanalytical and biophysical methods [9, 10]. Several offand on-line mass spectrometric (MS) methods have proven to be powerful tools for the characterization of metallodrugbiomolecule interactions [11]. Electrospray ionization (ESI) and MALDI have been used to study various systems including in vitro binding studies to both DNA and proteins $[8,12-14]$, and even whole cells were treated with cisplatin, digested, and analyzed $[15,16]$. The ability of metallodrugs to form adducts with plasma and cytoplasmic proteins such as HSA [8, 17, 18], myoglobin [19], transferrin [5, 20], model interactions with peptides [21, 22], and small model proteins such as ubiquitin (Ub) [11, 19, 23, 24], cytochrome-c (cyt-c) [25], lysozymes [7], and oligonucleotides $[22,26]$ have been subject of investigation by various MS methods.

In classical proteomics, bottom-up methodologies are widely used to characterize and identify protein sequences by analysis of enzymatic digests of proteins based on their mass fingerprints [27-29]. While bottom-up methods remain the best option for large proteins, they should cautiously be applied when investigating metallodrugprotein interactions since these may be prone to metallodrug cleavage during enzymatic digestion. More recently, top-down strategies were introduced [30], offering reduced experimental complexity and the ability to characterize entire protein sequences, whilst retaining post-translational modifications (PTMs) or even allowing the study of noncovalent interactions [31]. Considering metallation as a special type of PTM, top-down MS should be the method of choice for the detailed characterization of metallodrugbiomolecule conjugates [32]. Binding site localization can be achieved by tandem mass spectrometric methods, which include collision-induced dissociation (CID) [33], higherenergy C-trap dissociation (HCD) [34], infrared multiphoton dissociation [35], electron transfer dissociation (ETD) [36], or electron-capture dissociation (ECD) [37-39] and often deliver complementary information. CID and HCD are similar fragmentation methods relying on the collision of the molecules with inert gas molecules, which in turn leads to protein backbone cleavage, specifically at the amide $\mathrm{C}-\mathrm{N}$ bond to produce predominantly $b$ - and $y$ peptide fragments. Collisions with low-energy CID are less energetic than by HCD. Fragmentation by ECD and ETD is based on the transfer of electrons, which results in softer and more random protein backbone cleavages at $\mathrm{N}-\mathrm{C}_{\alpha}$ bonds and produces mainly $c$ - and $z$-type peptide fragments. These methods have rarely been employed in studies on metal-based anticancer agents and therefore, we report here a comparative study on the binding site characteriza- tion of oxaliplatin bound to Ub using different fragmentation methods in combination with high-resolution mass spectrometry. Oxaliplatin is the latest of the clinically approved platinum-based anticancer agents and compared with cisplatin, there are only a few investigations on binding interactions of this compound with biomolecules. The 76-amino acid protein Ub was established as a model in metallodrug binding studies $[11,19,23,24,40]$ and Met1 has been characterized as a binding partner for cisplatin [24] and His68 being believed to represent the second binding site.

\section{Experimental}

Materials and methods

Ub (from bovine red blood cells, min 90\%) was purchased from Sigma (Vienna, Austria), oxaliplatin from Sequoia Research Products (Pangbourne, UK), trifluoroacetic acid (98\%) from Fluka (Vienna, Austria), and methanol (HiPerSolv CHROMANORM) from VWR (Vienna, Austria). All materials from chemical suppliers were used as received. MilliQ water was obtained from a Millipore Synergy 185 UV Ultrapure apparatus (18.2 M $\Omega$ ). Oxaliplatin and $\mathrm{Ub}$ were incubated in a molar ratio of $2: 1$ in water $(\mathrm{pH} 5.5)$ for 7 days at $37{ }^{\circ} \mathrm{C}$. The reaction mixture was diluted with water/methanol/trifluoroacetic acid (50:50:0.1) to yield a final protein concentration of $1-5 \mu \mathrm{M}$, i.e., measurements were performed under denaturing conditions.

\section{Instrumentation}

LTQ Velos Orbitrap MS Incubation mixtures of oxaliplatin and $\mathrm{Ub}$ were introduced by direct infusion into the ESI source of the mass spectrometer at a flow rate of $5 \mu \mathrm{L} / \mathrm{min}$. Survey and tandem mass spectra were recorded on a hybrid LTQ Velos Orbitrap mass spectrometer (Thermo Scientific, Bremen, Germany) employing CID-, HCD-, and ETD-MS/ MS. Additional experimental settings were as follows: source voltage, $+4 \mathrm{kV}$; source temperature, $40{ }^{\circ} \mathrm{C}$; capillary temperature, $250{ }^{\circ} \mathrm{C}$; sheath gas flow, $10 \mathrm{~L} / \mathrm{min}$; minimal signal required, 500; and isolation width, $10 \mathrm{Th}$. The normalized collision energies during MS/MS experiments were $30 \%, 40 \%$, and $8 \%$ for CID, HCD, and ETD, respectively. Data were processed with Xcalibur 2.1 software (Thermo Scientific).

FT-ICR MS Incubation mixtures of oxaliplatin and Ub (ca. $3 \mu \mathrm{M}$ ) were introduced by direct infusion via an ESI source into a 9.4-T mass spectrometer (Bruker Daltonics GmbH, Bremen, Germany) with a flow rate of $0.5 \mu \mathrm{L} / \mathrm{min}$. CIDMS/MS measurements were performed with $10 \mathrm{~ms}$ accu- 
mulation time in the external quadrupole-based collisional cell. Additional instrument settings were as follows: capillary entrance voltage, $-1.5 \mathrm{kV}$; capillary exit voltage, $+100 \mathrm{~V}$; dry gas flow rate, $35 \mathrm{~L} / \mathrm{min}$; dry gas temperature, $200{ }^{\circ} \mathrm{C}$; scan width, $m / z$ 400-3,000; extraction voltage for ESI, $-10 \mathrm{~V}$; and nebulizing gas flow rate, $30 \mathrm{~L} / \mathrm{min}$. Spectra were acquired using Apex Control II. Data were analyzed and processed by ESI Compass 1.3 and Data Analysis 4.0 software (Bruker Daltonics).

\section{Results and discussion}

Characterization of oxaliplatin-Ub adducts by broadband ESI-MS

Oxaliplatin/Ub mixtures were incubated under non-denaturing conditions at $\mathrm{pH}$ 6, which reflect conditions that exist in hypoxic tumorigenic tissue [41, 42]. The incubation mixture was diluted to $1-5 \mu \mathrm{M}$ (protein) with $\mathrm{H}_{2} \mathrm{O} / \mathrm{MeOH} / \mathrm{TFA}$, causing denaturation of the protein to improve fragmentation efficiencies, and the samples were immediately injected into the ESI source of the mass spectrometer.

Platinum-based anticancer agents may interact with biomolecules at different stages of their activation process [43]. Oxaliplatin was designed to exhibit a very slow hydrolysis rate with a view to reduce unwanted side effects. Indeed, analysis of the reaction mixture containing a twofold excess of oxaliplatin by ESI-MS showed $\sim 50 \%$ free Ub after incubation for 7 days. The activation mechanism of oxaliplatin rendering it capable of binding to biological molecules is thought to involve two successive steps. Initially, one bond between platinum and the oxalato chelating ligand is hydrolyzed forming a mono-aqua complex, after which cleavage of the remaining platinumoxalato bond and ligand release from the platinum center occurs (Scheme 1). Therefore, several Pt-Ub derivatives were observed in the deconvoluted mass spectrum (Fig. 1) with the most prominent corresponding to an oxaliplatin-Ub adduct at $\mathrm{m} / \mathrm{z} 8,871.704$ ( $84 \%$ relative intensity to free Ub; all relative intensities refer to the most abundant peak in the respective mass spectrum). This signal can be assigned to a mono-adduct consisting of $\mathrm{Ub}$ and a Pt(chxn) moiety (chxn= $(1 R, 2 R)$-cyclohexanediamine) having undergone cleavage of the oxalato ligand. Further identified species include the mono-adduct after the first activation step $[\mathrm{Ub}+(\mathrm{ox}) \mathrm{Pt}$ $(\mathrm{chxn})]^{+}(\mathrm{m} / \mathrm{z}$ 8,961.697, 10\%; oxalate (ox)), as well as higher adducts such as $[\mathrm{Ub}+2 \mathrm{Pt}(\mathrm{chxn})]^{+}(\mathrm{m} / z$ 9,177.582, $24 \%)$ and $[\mathrm{Ub}+\mathrm{Pt}(\mathrm{chxn})+(\mathrm{ox}) \mathrm{Pt}(\mathrm{chxn})]^{+}(\mathrm{m} / \mathrm{z} \quad 9,268.751$, $11 \%$ ). The existence of platinum species stemming from the initial hydrolysis step was reported previously [43], and these findings provide further support for a two-step activation mechanism.

Comparative tandem mass spectrometry of oxaliplatin- $\mathrm{Ub}$ adducts

Top-down tandem mass spectrometric experiments on the incubation mixture were performed to directly identify binding sites of oxaliplatin on Ub using different fragmentation methods. These investigations were carried out with the most abundant adduct identified in the broadband mass spectrum, i.e., $[\mathrm{Ub}+\mathrm{Pt}(\mathrm{chxn})]$. The $12+$ charge state $(\mathrm{m} / \mathrm{z}$ 740.313) was selected for binding site characterization in the orbitrap FT MS-based experiments and, for comparison, the $11+$ charge stage $(\mathrm{m} / \mathrm{z}$ 807.515) in the FT-ICR MSbased experiments. The employment of charge states larger than $10+$ implies that $\mathrm{Ub}$ is entirely denatured and protonated to a large degree. CID-MS/MS was performed on both orbitrap- and ICR-FT MS instruments, whereas HCD- and ETD-MS/MS were performed on the orbitrap mass spectrometer only.

CID-MS/MS fragmentation profiles were comparable on both instruments and similar metallated peptide fragments were found. While the platinated peptide fragments were generally more abundant using FT-ICR MS, a higher number of such fragments was identified with the orbitrap MS approach. For the $[\mathrm{Ub}+\mathrm{Pt}(\mathrm{chxn})]$ adduct, CID led to the characterization of 23 different metallated peptide fragments for the orbitrap MS, compared to 15 with the FT-ICR MS. Assignable platinated peptide fragment ions

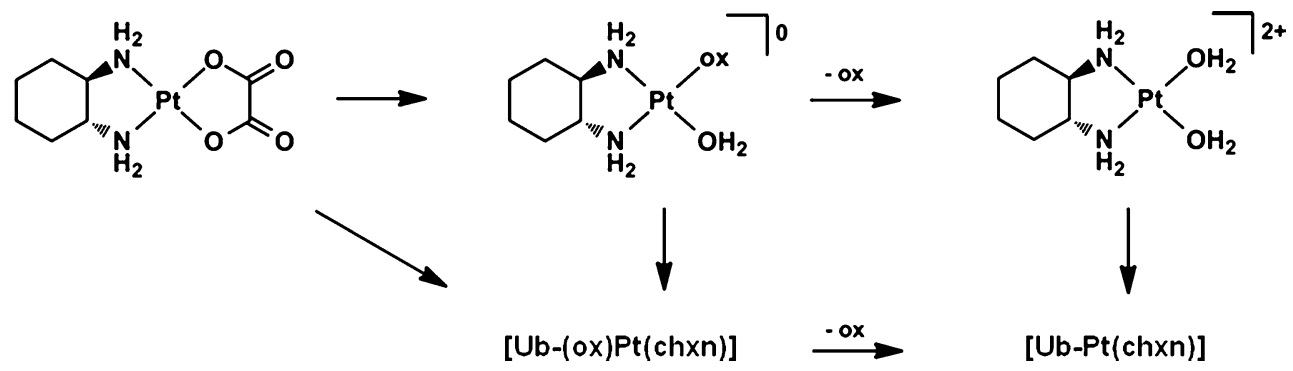

Scheme 1 Hydrolysis of oxaliplatin is responsible for activation of the prodrug and involves two successive steps (ox oxalate, chxn (1R,2R)cyclohexanediamine). Upon hydrolysis, oxaliplatin may more readily react with to suitable biological binding partners 


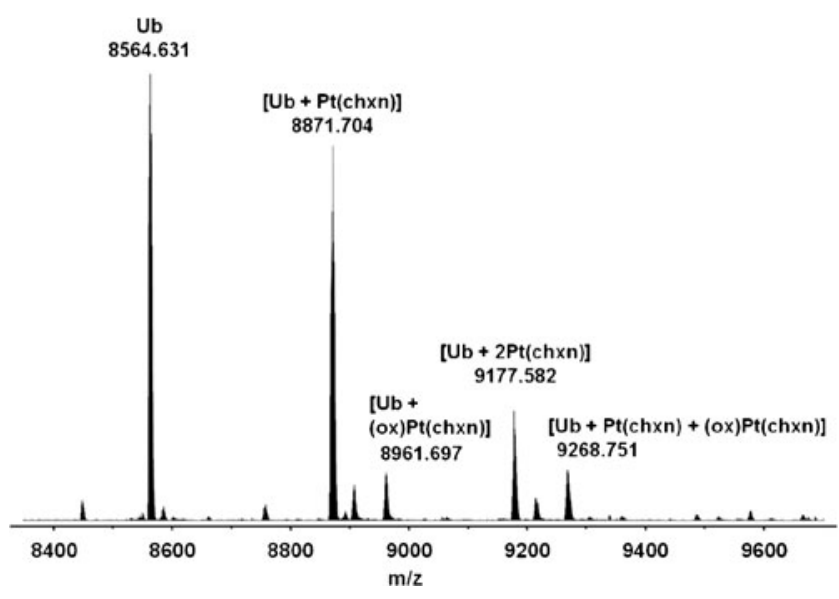

Fig. 1 Deconvoluted FT-ICR mass spectrum of the oxaliplatin/Ub incubation mixture after 7 days in the region $m / z \quad 8,400-9,600$. Oxaliplatin and $\mathrm{Ub}$ were reacted in a molar ratio of $2: 1$. The most abundant adduct corresponds to [Ub+Pt(chxn)]

were found over a broad mass (especially $m / z$ 700-1,000) and charge $(1+$ to $11+)$ range with a resolution of $R \approx 120,000$ at $m / z 567.171\left({ }^{\mathrm{Pt}(\mathrm{chxn})} \mathrm{B}_{2}{ }^{+}\right)$for both instrument types (Fig. 2). The mass accuracy of the metallated fragments was in general $<1 \mathrm{ppm}$.

Since bis-platinated $\mathrm{Ub}$ adducts were observed in significant intensities in the survey mass spectra, at least two main binding sites are to be expected for the $[\mathrm{Ub}+\mathrm{Pt}$ (chxn)] adducts. Indeed, the Pt(chxn)-containing peptide fragments detected by CID-MS/MS indicated two possible binding sites. The former was identified as $N$-terminal Met1 based on the ${ }^{\mathrm{Pt}(\mathrm{chxn})} \mathrm{B}_{2}{ }^{+}$ion (the following annotation system was used throughout the text to describe platinated peptide fragments: capital letters indicate metallated frag- ments, analogous to the conventional peptide ion type nomenclature. The nature of the bound metal species is given as preceding superscript; e.g., ${ }^{\mathrm{Pt}(\mathrm{chxn})} \mathrm{B}_{2}{ }^{+}$stands for the $\mathrm{Pt}(\mathrm{chxn})$ moiety bound to the $b_{2}$-fragment of $\mathrm{Ub}$ in charge state $1+$ ) as the smallest metal-containing peptide fragment, identified on both instruments and also observed earlier [23]. In addition, high molecular weight fragments, such as the $b_{2}$ complementary metallated fragment ${ }^{\mathrm{Pt}}$ ${ }^{(\mathrm{chxn})} \mathrm{Y}_{74}{ }^{10+/ 11+}$, indicate the presence of a second binding site. Several C-terminal metallated peptide fragments were detected with both instruments. They range from ${ }^{\mathrm{Pt}}$ ${ }^{(\text {chxn })} \mathrm{Y}_{24}{ }^{5+}$ to ${ }^{\mathrm{Pt}(\mathrm{chxn})} \mathrm{Y}_{75}{ }^{11+}$ on the orbitrap FT MS and from ${ }^{\mathrm{Pt}(\mathrm{chxn})} \mathrm{Y}_{18}{ }^{3+}$ to ${ }^{\mathrm{Pt}(\mathrm{chxn})} \mathrm{Y}_{74}{ }^{10+}$ on the FT-ICR MS. These fragments do not give the exact location of the second binding site, but they do reveal that it must be within the last 18 residues of the $\mathrm{C}$-terminal protein sequence. Most of the B- and Y-fragments are centered around $y_{58}$, which was observed in all collisionally activated tandem mass spectra, i.e., ${ }^{\mathrm{Pt}(\mathrm{chxn})} \mathrm{B}_{16}{ }^{2+}$, and also the complementary peptide fragment $y_{60}{ }^{8+}(\mathrm{m} / \mathrm{z}$ 845.833, $1 \%$ ) was detected.

HCD is a fragmentation technique that makes use of higher-energy collisions and generally results in increased fragmentation efficiency and sequence coverage. Indeed, 27 metallated fragments were unambiguously identified in the HCD-orbitrap MS/MS experiments. In addition, higherenergy collisions yielded a fragmentation profile where the metallated peptide fragments were generally smaller and of lower charge than with CID-orbitrap MS/MS. N- and Cterminal metallated fragments were essentially equal in intensity and number. HCD gave rise to metallodrugpeptide fragments with higher relative intensities compared with CID and with similar resolution and mass accuracies of $<1$ ppm (Fig. 3a).

Fig. 2 (a) CID-orbitrap- and (b) CID-ICR-FT tandem mass spectra of the [Ub+Pt(chxn)] adduct. Oxaliplatin and $\mathrm{Ub}$ were reacted in 2:1 molar ratio and incubated for 7 days. The two most probable binding partners, i.e., Met1 and His68, are underlined in the amino acid sequence of Ub. Bars between the sequence letters refer to metallated peptide fragments




Fig. 3 (a) HCD-orbitrap- and (b) ETD-orbitrap tandem mass spectra of the [Ub+Pt(chxn)] adduct. Oxaliplatin and $\mathrm{Ub}$ were reacted in 2:1 molar ratio and incubated for 7 days. The two most probable binding partners, i.e., Met1 and His68, are underlined in the amino acid sequence of Ub. Solid bars between the sequence letters refer to detected metallated peptide fragments whereas the dashed bars refer to the $y_{8}$ and $z_{8}$ fragments

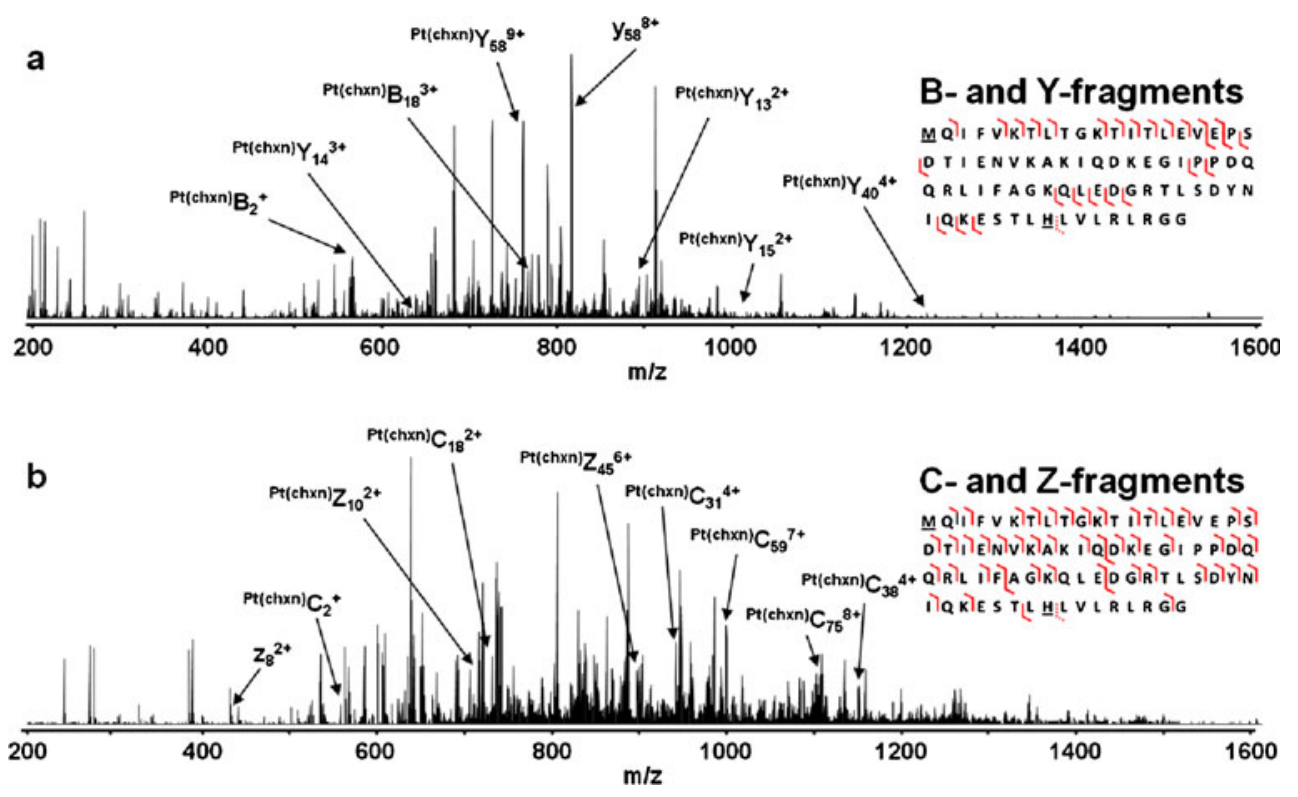

Many metallated $N$-terminal fragments were detected, ranging from ${ }^{\mathrm{Pt}(\mathrm{chxn})} \mathrm{B}_{2}{ }^{+}$to ${ }^{\mathrm{Pt}(\mathrm{chxn})} \mathrm{B}_{18}{ }^{3+}$, also confirming Met1 as the primary binding site for oxaliplatin. With regard to the second binding site of oxaliplatin, small platinated and non-platinated C-terminal fragments were analyzed. Indeed, the HCD tandem mass spectrum contained signals assignable to non-metallated $y_{8}{ }^{2+}$ ions, as well as metallated ${ }^{\mathrm{Pt}(\mathrm{chxn})} \mathrm{Y}_{13}{ }^{2+},{ }^{\mathrm{Pt}(\mathrm{chxn})} \mathrm{Y}_{14}{ }^{2+}$ and ${ }^{\mathrm{Pt}(\mathrm{chxn})} \mathrm{Y}_{15}{ }^{2+}$ fragments. This finding narrowed the number of possible binding sites to a 5-amino acid sequence containing Glu64 and His68 as most nucleophilic sites. In contrast to CID where complementary fragments were found at several points along the protein sequence, the only observable complementary pair was the ${ }^{\mathrm{Pt}(\mathrm{chxn})} \mathrm{B}_{18}{ }^{3+} / y_{58}{ }^{8+}$ couple.

In parallel to collision-induced fragmentation techniques, the $[\mathrm{Ub}+\mathrm{Pt}(\mathrm{chxn})]$ adduct was subjected to ETD. This fragmentation method, based on ion/ion reactions resulting in electron transfers, leads to $\mathrm{N}-\mathrm{C}_{\alpha}$ bond breaks along the peptide backbone and specifically yields $c$ - and $z$-fragment ions. The general advantage of ETD is that it retains PTMs to a large extent, making it a valuable tool for top-down protein sequencing in proteomics. In addition, electron transfer should result in a more random fragmentation pattern relative to collisionally induced fragmentation techniques.

ETD-MS/MS generated a very different fragmentation profile in comparison to collisionally activated fragmentation methods (Fig. 3b). Even though the relative intensities and the resolution $\left(R \approx 120,000\right.$ at $\left.\mathrm{m} / z 586.785\left[{ }^{\mathrm{Pt}(\mathrm{chxn})} \mathrm{C}_{7}{ }^{2+}\right]\right)$ of the platinated peptide fragments were similar to those obtained by CID and HCD methods, ETD allowed identifying a higher number of metallated fragments, i.e., 50 in total, which represents the highest value among the different fragmentation techniques. A total of $46 \mathrm{~N}$-terminal platinated fragments were detected ranging from ${ }^{\mathrm{Pt}(\mathrm{chxn})} \mathrm{C}_{2}{ }^{+}$to ${ }^{\mathrm{Pt}(\mathrm{chxn})} \mathrm{C}_{75}{ }^{8+}$, thus verifying Met1 as the first binding partner of oxaliplatin on $\mathrm{Ub}$. The most abundant metal-containing fragment was identified as ${ }^{\mathrm{Pt}(\mathrm{chxn})} \mathrm{C}_{59}{ }^{7+}(21 \%)$, as opposed to ${ }^{\mathrm{Pt}(\mathrm{chxn})} \mathrm{B}_{18}{ }^{2+/ 3+}$ for collisionally activated methods, which may be related to the different fragmentation mechanisms. It is believed that positively charged amino acids act as electron scavengers during electron transfer [44]. In the case of $\mathrm{Ub}$, Lys48 is in close proximity to the peptide bond between Tyr59 and Asn60. This may facilitate backbone cleavage and be responsible for the high abundance observed. In addition, collisionally induced fragmentation at Glu18 may also produce very stable products, i.e., in source generation of $y_{58}$ is known. On the other hand, Cterminal metallated peptide fragments ranged from ${ }^{\mathrm{Pt}(\mathrm{chxn})} \mathrm{Z}_{10}{ }^{2+}$ to ${ }^{\mathrm{Pt}(\mathrm{chxn})} \mathrm{Z}_{45}{ }^{6+}$. The metallated ${ }^{\mathrm{Pt}(\mathrm{ch} x \mathrm{x})} \mathrm{Z}_{10}{ }^{2+}$ and non-metallated $z_{8}{ }^{2+} \mathrm{C}$-terminal fragments clearly identify the second binding site as His68 excluding Glu64 as a potential binding partner. ETD therefore emerges as a superior fragmentation technique to CID and HCD when investigating metallodrug-protein adducts via a top-down approach both in terms of fragment numbers and localization of binding sites. It is the only fragmentation technique that allowed the simultaneous identification of both Met1 and His68 as binding partners from a single precursor. The lower efficiency of CID- and HCD-MS/MS for detecting metallated peptides might be due to collision-induced gas phase demetallation, whereas ETD-MS/MS is a softer fragmentation technique and might retain coordinative Pt-protein bonds to a larger degree.

A striking observation is the large discrepancy between the number of C- and Z-fragments (46:4). One explanation 
might involve favored binding of oxaliplatin to the Nterminal Met1 which causes a higher abundance of such fragments compared with C-terminal His68. In support of this hypothesis is the fact that the number of characterized $z$-fragments during ETD measurements on bare $\mathrm{Ub}$ is approximately three times higher than the respective $c$ fragments. Therefore, Met1 is considered the primary binding site of oxaliplatin on Ub.

Insights into the secondary structure of the $[\mathrm{Ub}+\mathrm{Pt}(\operatorname{chxn})]$ adduct

ESI mass spectrometry is extensively used to gain information on the higher order structure of proteins, in particular in the elucidation of the tertiary structure. For this purpose, several approaches have proven effective, the most widespread being H/D-exchange [45, 46], covalent labeling [47], and ion mobility MS $[24,48]$. The tertiary protein structure is affected by the solvent conditions employed during incubation and infusion into the mass spectrometer [49, 50]. Importantly, a correlation between charge state (i.e., unfolding), temperature and fragmentation efficiency has been established [51]. In contrast to tertiary structure, only a few experiments were conducted on protein secondary structures by means of ESI mass spectrometry. Secondary structures are supposed to remain, to some degree, intact under denaturing conditions but it is unknown if the same secondary structures are retained in the gas phase as present in solution [49, 51-54].

In the present study, MS/MS experiments were performed under denaturing conditions which led to breakdown of the protein tertiary structure. When the protein transfers from solution to the gas phase at the ESI interface, hydrophobic interactions are further diminished and electrostatic effects enhanced, resulting in the protein adopting a near-linear conformation [51]. Secondary structures are stabilized by H-bonding in the gas phase and presumably more energy is required in collisionally activated fragmentation experiments to cleave the protein backbone at these locations compared to random coil sequences. Interestingly, plotting the MS/MS-fragment intensities from the combined CID and HCD data sets relative to the sequence revealed a strong correlation with solution random coil structures (Fig. 4) [55]. The backbone cleavages suggest that the Nterminal $\beta$-hairpin section stays intact. The random coil sequence Val17-Thr22 connects the $\beta$-hairpin with the only $\alpha$-helix in Ub and from Glu16-Ile23, six out of eight peptide bond cleavages were observed. The section of the $\alpha$-helix remained intact to a large degree. The next cleavage occurred prior to the successive prolines at positions $36 / 37$, which initiate the $\alpha$-turn. The subsequent $\beta$-strand is followed by an extended random coil sequence, which displays four consecutive cleavages from Gln49-Asp52. The remaining $\alpha$-turn and $\beta$-strand sections are not sufficiently stabilized when the protein adopts a near-linear conformation and therefore, numerous cleavages occur between Asp58 and Glu64. These results suggest that solution secondary structures of the [Ub+Pt(chxn)] adduct are retained to a considerable extent in the gas phase. Contrary to CID and HCD methods, ETD did not yield such pronounced specificity because it generates more random cleavages on the protein backbone (Fig. 4).

\section{Conclusions}

This work highlights the application of high-resolution mass spectrometric methods for top-down characterization of metallodrug-protein adducts. The major adduct of oxaliplatin at $\mathrm{Ub}$ is formed with a $\mathrm{Pt}(\mathrm{chxn})$ moiety. In addition, the experimental setup yielded further adduct types, which were identified as $[\mathrm{Ub}+(\mathrm{ox}) \mathrm{Pt}(\mathrm{chxn})],[\mathrm{Ub}+$ $2 \mathrm{Pt}(\operatorname{chxn})]$, and $[\mathrm{Ub}+\mathrm{Pt}(\operatorname{chxn})+(\mathrm{ox}) \mathrm{Pt}(\mathrm{chxn})]$ in support of a two-step activation mechanism of oxaliplatin. ESI Orbitrap- and ICR-FT MS combined with CID-, HCD-, and ETD-MS/MS data were used to locate the binding sites of oxaliplatin on $\mathrm{Ub}$. All fragmentation experiments were carried out under denaturing conditions and analyzed in high charge states in order to maximize fragmentation efficiency. ETD yielded the highest number of metallated

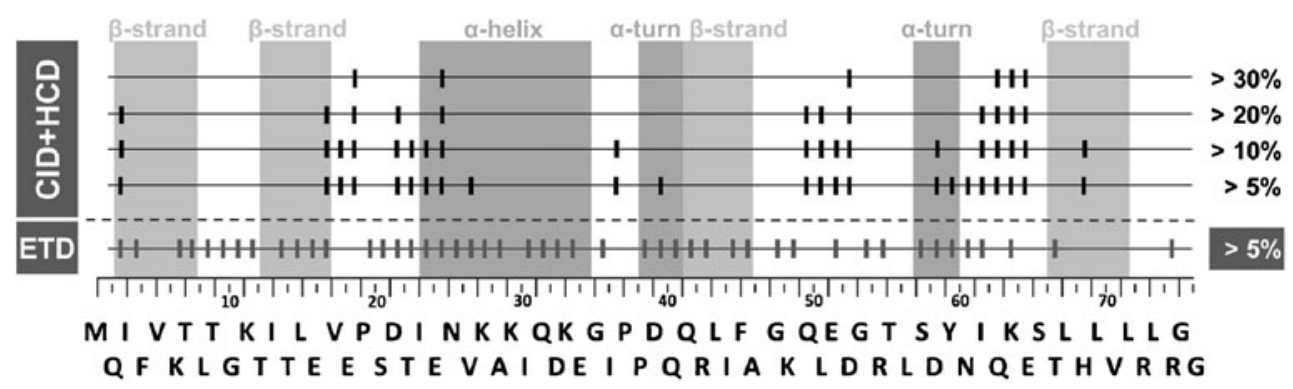

Fig. 4 A combined plot of backbone cleavage sites (bars) depending on decreasing relative intensities observed during CID- and HCD-MS/ MS experiments with the $[\mathrm{Ub}+\mathrm{Pt}(\mathrm{chxn})]$ adduct. The last row features cleavages from the ETD-MS/MS experiment. The positions of the secondary structures were determined by the Kabsch-Sanders algorithm. White background refers to random coil sequence areas 
fragments and was the only technique to unequivocally establish Met1 and His68 as the two binding sites for oxaliplatin on Ub. From this study, ETD has emerged as a powerful method for characterizing metallodrug-protein binding sites. On the other hand only collisionally activated methods yielded insights into the secondary structure of Ub. In particular, a correlation was found between peptide fragments and cleavage at random coil sequences, indicating that $\beta$-hairpin and $\alpha$-helix structures of the $[\mathrm{Ub}+\mathrm{Pt}$ (chxn)] adduct are retained in the gas phase.

Acknowledgment The authors are indebted to the Austrian Science Fund (FWF; I496-B11), the Hochschuljubiläumsstiftung Vienna and COST D39 and CM0902. We would like to thank Dr. Yue Xuan (Thermo Scientific) and Dr. Jens Fuchser (Bruker Daltonics) for assistance during measurements on the orbitrap FT MS and FT-ICR MS instruments, respectively.

\section{References}

1. Barry MA, Behnke CA, Eastman A (1990) Activation of programmed cell-death (apoptosis) by cisplatin, other anticancer drugs, toxins and hyperthermia. Biochem Pharmacol 40:23532362

2. Ivanov AI, Christodoulou J, Parkinson JA, Barnham KJ, Tucker A, Woodrow J, Sadler PJ (1998) Cisplatin binding sites on human albumin. J Biol Chem 273:14721-14730

3. Groessl M, Zava O, Dyson PJ (2011) Cellular uptake and subcellular distribution of ruthenium-based metallodrugs under clinical investigation versus cisplatin. Metallomics 3:591599

4. Lokich J, Anderson N (1998) Carboplatin versus cisplatin in solid tumors: an analysis of the literature. Ann Oncol 9:13-21

5. Allardyce CS, Dyson PJ, Coffey J, Johnson N (2002) Determination of drug binding sites to proteins by electrospray ionisation mass spectrometry: the interaction of cisplatin with transferrin. Rapid Commun Mass Spectrom 16:933-935

6. Calderone V, Casini A, Mangani S, Messori L, Orioli PL (2006) Structural investigation of cisplatin-protein interactions: selective platination of His19 in a cuprozinc superoxide dismutase. Angew Chem Int Ed Engl 45:1267-1269

7. Casini A, Mastrobuoni G, Temperini C, Gabbiani C, Francese S, Moneti G, Supuran CT, Scozzafava A, Messori L (2007) ESI mass spectrometry and X-ray diffraction studies of adducts between anticancer platinum drugs and hen egg white lysozyme. Chem Commun (2):156-158

8. Groessl M, Terenghi M, Casini A, Elviri L, Lobinski R, Dyson PJ (2010) Reactivity of anticancer metallodrugs with serum proteins: new insights from size exclusion chromatography-ICP-MS and ESI-MS. J Anal At Spectrom 25:305-313

9. Timerbaev AR, Hartinger CG, Aleksenko SS, Keppler BK (2006) Interactions of antitumor metallodrugs with serum proteins: advances in characterization using modern analytical methodology. Chem Rev 106:2224-2248

10. Sun X, Tsang C-N, Sun H (2009) Identification and characterization of metallodrug binding proteins by (metallo)proteomics. Metallomics 1:25-31

11. Hartinger CG, Ang WH, Casini A, Messori L, Keppler BK, Dyson PJ (2007) Mass spectrometric analysis of ubiquitinplatinum interactions of leading anticancer drugs: MALDI versus ESI. J Anal At Spectrom 22:960-967
12. Hartinger CG, Casini A, Duhot C, Tsybin YO, Messori L, Dyson PJ (2008) Stability of an organometallic ruthenium-ubiquitin adduct in the presence of glutathione: relevance to antitumor activity. J Inorg Biochem 102:2136-2141

13. Ang WH, Parker LJ, De Luca A, Juillerat-Jeanneret L, Morton CJ, Lo Bello M, Parker MW, Dyson PJ (2009) Rational design of an organometallic glutathione transferase inhibitor. Angew Chem Int Ed 48:3854-3857

14. Egger AE, Hartinger CG, Renfrew AK, Dyson PJ (2010) Metabolization of $\left[\mathrm{Ru}\left(\eta^{6}-\mathrm{C}_{6} \mathrm{H}_{5} \mathrm{CF}_{3}\right)(\mathrm{pta}) \mathrm{Cl}_{2}\right]$ : a cytotoxic RAPTA-type complex with a strongly electron withdrawing arene ligand. J Biol Inorg Chem 15:919-927

15. Allardyce CS, Dyson PJ, Abou-Shakra FR, Birtwistle H, Coffey J (2001) Inductively coupled plasma mass spectrometry to identify protein drug targets from whole cell systems. Chem Commun 2708-2709.

16. Scharwitz MA, Ott I, Geldmacher Y, Gust R, Sheldrick WS (2008) Cytotoxic half-sandwich rhodium(III) complexes: polypyridyl ligand influence on their DNA binding properties and cellular uptake. J Organomet Chem 693:2299-2309

17. Timerbaev AR, Aleksenko KS, Polec-Pawlak K, Ruzik R, Semenova O, Hartinger CG, Oszwaldowski S, Galanski M, Jarosz M, Keppler BK (2004) Platinum metallodrug-protein binding studies by capillary electrophoresis-inductively coupled plasmamass spectrometry: characterization of interactions between $\mathrm{Pt}(\mathrm{II})$ complexes and human serum albumin. Electrophoresis 25:19881995

18. Will J, Wolters DA, Sheldrick WS (2008) Characterisation of cisplatin binding sites in human serum proteins using hyphenated multidimensional liquid chromatography and ESI tandem mass spectrometry. ChemMedChem 3:1696-1707

19. Najajreh Y, Shulman TP, Moshel O, Farrell N, Gibson D (2003) Ligand effects on the binding of cis- and trans-[PtCl(2)Am1 Am2] to proteins. J Biol Inorg Chem 8:167-175

20. Khalaila I, Allardyce CS, Verma CS, Dyson PJ (2005) A mass spectrometric and molecular modelling study of cisplatin binding to transferrin. Chembiochem 6:1788-1795

21. Williams JP, Brown JM, Campuzano I, Sadler PJ (2010) Identifying drug metallation sites on peptides using electron transfer dissociation (ETD), collision induced dissociation (CID) and ion mobility-mass spectrometry (IM-MS). Chem Commun 46:5458-5460

22. Groessl M, Tsybin YO, Hartinger CG, Keppler BK, Dyson PJ (2010) Ruthenium versus platinum: interactions of anticancer metallodrugs with duplex oligonucleotides characterised by electrospray ionisation mass spectrometry. J Biol Inorg Chem 15:677-688

23. Hartinger CG, Tsybin YO, Fuchser J, Dyson PJ (2008) Characterization of platinum anticancer drug protein-binding sites using a top-down mass spectrometric approach. Inorg Chem 47:17-19

24. Williams JP, Phillips HIA, Campuzano I, Sadler PJ (2010) Shape changes induced by $\mathrm{N}$-terminal platination of ubiquitin by cisplatin. J Am Soc Mass Spectrom 21:1097-1106

25. Casini A, Guerri A, Gabbiani C, Messori L (2008) Biophysical characterisation of adducts formed between anticancer metallodrugs and selected proteins: new insights from X-ray diffraction and mass spectrometry studies. J Inorg Biochem 102:995-1006

26. Krivos KL, Limbach PA (2010) Sequence analysis of peptide: oligonucleotide heteroconjugates by electron capture dissociation and electron transfer dissociation. J Am Soc Mass Spectrom 21:1387-1397

27. van der Rest G, He F, Emmett MR, Marshall AG, Gaskell SJ (2001) Gas-phase cleavage of PTC-derivatized electrosprayed tryptic peptides in an FT-ICR trapped-ion cell: mass-based protein identification without liquid chromatographic separation. J Am Soc Mass Spectrom 12:288-295 
28. Han XM, Aslanian A, Yates JR (2008) Mass spectrometry for proteomics. Curr Opin Chem Biol 12:483-490

29. Zhao T, King FL (2011) Mass-spectrometric characterization of cisplatin binding sites on native and denatured ubiquitin. J Biol Inorg Chem 16:633-639

30. Kelleher NL (2004) Top-down proteomics. Anal Chem 76:196a203a

31. Yin S, Loo JA (2010) Elucidating the site of protein-ATP binding by top-down mass spectrometry. J Am Soc Mass Spectrom 21:899-907

32. Moreno-Gordaliza E, Canas B, Palacios MA, Gomez-Gomez MM (2009) Top-down mass spectrometric approach for the full characterization of insulin-cisplatin adducts. Anal Chem 81:3507-3516

33. Papayannopoulos IA (1995) The interpretation of collisioninduced dissociation tandem mass-spectra of peptides. Mass Spectrom Rev 14:49-73

34. Olsen JV, Macek B, Lange O, Makarov A, Horning S, Mann M (2007) Higher-energy C-trap dissociation for peptide modification analysis. Nat Methods 4:709-712

35. Laskin J, Futrell JH (2005) Activation of large ions in FT-ICR mass spectrometry. Mass Spectrom Rev 24:135-167

36. Mikesh LM, Ueberheide B, Chi A, Coon JJ, Syka JE, Shabanowitz J, Hunt DF (2006) The utility of ETD mass spectrometry in proteomic analysis. Biochim Biophys Acta 1764:1811-1822

37. Nielsen ML, Savitski MM, Zubarev RA (2005) Improving protein identification using complementary fragmentation techniques in Fourier transform mass spectrometry. Mol Cell Proteomics 4:835845

38. Syrstad EA, Turecek F (2005) Toward a general mechanism of electron capture dissociation. J Am Soc Mass Spectrom 16:208-224

39. Zubarev RA, Zubarev AR, Savitski MM (2008) Electron capture/ transfer versus collisionally activated/induced dissociations: solo or duet? J Am Soc Mass Spectrom 19:753-761

40. Peleg-Shulman T, Gibson D (2001) Cisplatin-protein adducts are efficiently removed by glutathione but not by 5 -guanosine monophosphate. J Am Chem Soc 123:3171-3172

41. Hartinger CG, Schluga P, Galanski M, Baumgartner C, Timerbaev AR, Keppler BK (2003) Tumor-inhibiting platinum(II) complexes with aminoalcohol ligands: comparison of the mode of action by capillary electrophoresis and electrospray ionization-mass spectrometry. Electrophoresis 24:2038-2044

42. Galanski M, Baumgartner C, Meelich K, Arion VB, Fremuth M, Jakupec MA, Schluga P, Hartinger CG, Von Keyserlingk NG, Keppler BK (2004) Synthesis, crystal structure and pH dependent cytotoxicity of (SP-4-2)-bis(2-aminoethanolato- $\mathrm{K}^{2} \mathrm{~N}, O$ ) platinum (II) - a representative of novel $\mathrm{pH}$ sensitive anticancer platinum complexes. Inorg Chim Acta 357:3237-3244
43. Jerremalm E, Videhult P, Alvelius G, Griffiths WJ, Bergman T, Eksborg S, Ehrsson H (2002) Alkaline hydrolysis of oxaliplatinisolation and identification of the oxalato monodentate intermediate. J Pharm Sci 91:2116-2121

44. Simons J (2010) Mechanisms for S-S and N-C-alpha bond cleavage in peptide ECD and ETD mass spectrometry. Chem Phys Lett 484:81-95

45. Briggs MS, Roder H (1992) Early hydrogen-bonding events in the folding reaction of ubiquitin. Proc Natl Acad Sci 89:2017-2021

46. Pan J, Han J, Borchers CH, Konermann L (2008) Electron capture dissociation of electrosprayed protein ions for spatially resolved hydrogen exchange measurements. J Am Chem Soc 130:1157411575

47. Mendoza VL, Vachet RW (2009) Probing protein structure by amino acid-specific covalent labeling and mass spectrometry. Mass Spectrom Rev 28:785-815

48. Robinson EW, Leib RD, Williams ER (2006) The role of conformation on electron capture dissociation of ubiquitin. J Am Soc Mass Spectrom 17:1469-1479

49. Konermann L, Douglas DJ (1997) Acid-induced unfolding of cytochrome $\mathrm{c}$ at different methanol concentrations: electrospray ionization mass spectrometry specifically monitors changes in the tertiary structure. Biochemistry 36:12296-12302

50. Konermann L, Douglas DJ (1998) Equilibrium unfolding of proteins monitored by electrospray ionization mass spectrometry: Distinguishing two-state from multi-state transitions. Rapid Commun Mass Spectrom 12:435-442

51. Breuker K, Oh HB, Horn DM, Cerda BA, McLafferty FW (2002) Detailed unfolding and folding of gaseous ubiquitin ions characterized by electron capture dissociation. J Am Chem Soc 124:6407-6420

52. Oh H, Breuker K, Sze SK, Ge Y, Carpenter BK, McLafferty FW (2002) Secondary and tertiary structures of gaseous protein ions characterized by electron capture dissociation mass spectrometry and photofragment spectroscopy. Proc Natl Acad Sci 99:1586315868

53. Ben Hamidane H, He H, Tsybin OY, Emmett MR, Hendrickson CL, Marshall AG, Tsybin YO (2009) Periodic sequence distribution of product ion abundances in electron capture dissociation of amphipathic peptides and proteins. J Am Soc Mass Spectrom 20:1182-1192

54. Breuker K, Bruschweiler S, Tollinger M (2011) Electrostatic stabilization of a native protein structure in the gas phase. Angew Chem Int Ed Engl 50:873-877

55. Zhang Z, Bordas-Nagy J (2006) Peptide conformation in gas phase probed by collision-induced dissociation and its correlation to conformation in condensed phases. J Am Soc Mass Spectrom 17:786-794 